\title{
FACTORES QUE INFLUYEN EN EL DESARROLLO DE PROYECTOS EN INSTITUCIONES PÚBLICAS
}

Factors influencing the development of projects in public institutions

\section{Raúl Eduardo Reyes Ramírez}

Maestro en Gestión Pública, Universidad César Vallejo, Lima, Perú, Ing.reyesramirez@gmail.com, ORCID: 0000-0002-0993-0568,

https://scholar.google.es/citations?hl=es\&user=FW2WIPoAAAAJ

\section{Deborah Mercedes Castro Castillo}

Maestra en Gestión Pública, Universidad César Vallejo, Lima, Perú demeca79@gmail.com ORCID: 0000-0001-5941-2876

https://scholar.google.com/citations?user=csfDRrUAAAAJ\&hl=es\&authuser=1

\section{Díaz Espinoza, Maribel}

Doctora en Administración de la Educación, Universidad Tecnológica del Perú, Piura, Perú c20937@utp.edu.pe ORCID: 0000-0001-5208-8380

https://scholar.google.com/citations?user=uI7T9DUAAAAJ\&hl=es

\section{Zapata Quispe, Raquel}

Maestro en Gestión Pública y Gobernabilidad, Universidad César Vallejo raquelzq1@gmail.com ORCID 0000-0002-4694-8417

https://scholar.google.com/citations?hl=es\&user=qawF8U0AAAAJ

Revista Iberoamericana de la Educación

Vol - Especial 12021

e-ISSN: 2737-632x

Resumen: El presente artículo aborda el desarrollo de alternativas respondiendo a necesidades de colectivos humanos, los objetivos se inspiran en un trabajo de investigación en desarrollo que persigue identificar los elementos de diseño de un proyecto especial, diagnosticar los factores que inciden en la ejecución de proyectos especiales, determinar los factores para la mejora de estos proyectos, en este sentido, la respuesta estratégica para identificar las necesidades y su aplicación a carencias como la salud, educación, empleo, acceso a la vivienda y servicios eléctricos, y sanitarios que afectan a los ciudadanos, Munsibay (2019), Armendáriz \& Fretes, (2017), Lovón (2019), entre otros, destacan la existencia de necesidades y 
cómo los proyectos aporten respuestas para la población, la metodología es investigación empírica, como fenómeno contemporáneo en entorno real con informantes de la gestión de proyectos especiales, analizando creación y gestión. Los resultados estarán orientados por Project Managment Institute (PMI), zonas de conocimiento, debatiendo la desviación de costos, tiempo en la ejecución, calidad de logro, llegando a conclusiones y recomendaciones vinculadas a estos bajo los lineamientos de la teoría general de sistemas cuyo enfoque profundiza las características a nivel estructural y funcional de los proyectos como sistemas activos, flexibles y auditables.

Palabras clave: Proyectos especiales- sistemas activos - respuesta estratégica

Abastract: This study addresses the development of alternatives responding to the needs of human groups, the objectives are inspired by a research work in development that seeks to identify the design elements of a special project, diagnose the factors that affect the execution of special projects, determine the factors for the improvement of these projects, in this sense, the strategic response to identify the needs and their application to deficiencies such as health, education, employment, access to housing and electrical and sanitary services that affect citizens, Munsibay (2019), Armendáriz \& Fretes, (2017), Lovón (2019), among others, highlight the existence of needs and how the projects provide answers for the population, the methodology is empirical research, as a contemporary phenomenon in a real environment with informants of the management of special projects, analyzing creation and management. The results will be guided by Project Management Institute (PMI), knowledge areas, debating cost deviation, time in execution, quality of achievement, reaching conclusions and recommendations related to these under the guidelines of the general theory of systems whose approach deepens 
the structural and functional characteristics of projects as active, flexible and auditable systems.

Keywords: Special projects - active systems - Estrategic response

\section{INTRODUCCIÓN}

Se ha apreciado que en la puesta en práctica de los proyectos de inversión pública a nivel social, la tarea no concluye con la determinación del impacto de dichos proyectos, a este respecto, Munsibay (2019), destaca que este cierre es cuando ya puestos en operación se valore su influencia en la calidad de vida de la población. Pues según Armendáriz \& Fretes, (2017), existe la necesidad de que los proyectos como logros arrojen que toda la población disponga de bienes y servicios idóneos, que redunden en la calidad de vida, y esto es capaz de realizarse a través de ejecución de inversiones, a través de evaluación de criterios, resultados y de eficiencia, así como, de gobernabilidad de las instituciones públicas, pues se presentan una serie de acciones que apuntan a la mejora de la gestión.

Es por ello, que dada la importancia de la gestión desde una visión práctica aportar a identificar los factores que inciden negativamente y positivamente en la de esta desde los aspectos de la optimización del sistema de gestión y control, permitiendo recomendar soluciones a las diversas problemáticas del conglomerado social, el cual necesita respuestas en cuanto a salud, educación, servicios público, tecnológicos y de tránsito a nivel urbano y rural, siendo útil para establecer un visión a futuro el planificar proyectos en los niveles decisorios a nivel nacional y distrital, este enfoque se hace en función de las opiniones de expertos, cuyas referencias internacionales y nacionales relacionadas a la gestión de proyectos desde una visión de plantear los procesos inherentes a esta construcción. Asimismo, esta modalidad de planeación y ejecución se han denominado proyectos especiales, los cuales 
están normados en Perú por los fundamentos de la Ley $\mathrm{N}^{\circ} 27654$ Marco de Modernización de la Gestión del Estado, se espera que los aporte brindados en este artículo aportarán al fortalecimiento del Estado en la mejora de la calidad en el servicio al ciudadano y por ende la gestión pública.

El objetivo planteado bajo el contexto propuesto es analizar los proyectos especiales para dar satisfacción a las necesidades de la población a través del cierre de brechas o valor público, cuya finalidad es formular propuestas en ámbitos de salud, educación y sociales con el fin de dar respuesta para mejorar las gestiones orientadas con metodologías que incluyan organización, dirección, coordinación, planificación, control, evaluación y otras estrategias que apoyen el acceso a recursos materiales y talentos humanos que guíen el alcance de metas a corto, mediano y largo plazo, considerando los elementos de diseño, los pasos para la ejecución, los factores que inciden en ella y dictaminen espacios de flexibilidad y realimentación para desde la evaluación alcanzar las propuestas.

Los proyectos, según el aporte de Lovón (2019), tienen diversos factores asociados, desde su concepción y muchos de estos inciden en la ejecución, por lo cual es importante establecer y analizar las distintas causas que influyen en su elaboración, como base para entender la gestión administrativa, la cual permite desde el enfoque cualitativo y diseño fenomenológico, analizar desde el nivel de preparación y experiencia del talento humano que los diseñan y ejecutan, relacionando el conocimiento del personal con los niveles de logro del proyecto de inversión pública, se considera que la problemática se centra en la etapa de concepción del proyecto, de allí que los objetivos que dan origen a este artículo son identificar los elementos de diseño de proyectos especiales que expliquen su ejecución, diagnosticar los factores que inciden en la ejecución y los factores para la mejora de la situación o necesidad planteada. 
Por otra parte, es importante definir lo que se entiende por proyecto, según Baca y Herrera (2016) son la manifestación técnica para brindar soluciones a las problemáticas de la sociedad, lo que redunda en el beneficio para las personas que requieren esta respuesta. Destaca Vera, L (2015) que la intervención del Estado en los proyectos, los define como proyectos de inversión pública, eso implica que los recursos provienen del Estado con el fin de generar beneficios a la sociedad, tomando en cuenta lo que según Palchisaca, M. (2015), define el éxito logrado, que se relaciona con la integración de las áreas de presupuesto y planificación, de allí que Sanz, A. (2015), destaca como punto clave la alineación del presupuesto con el plan, lo que implica una sana gestión de finanzas.

En el presente artículo se ha realizado una revisión de la literatura para sistematizar las bases del pensamiento orientado a algunos aspectos de interés, entre ellos Aranzamendi (2017) estableció que las mejoras en los procesos de proyectos basados en la integración, alcance, tiempo, costo, calidad, recursos humanos, comunicaciones y riesgos, arrojan que falta mejorar los indicadores anteriores, aspecto compartido por Mattar, J. y Cuervo, L. (2017), al considerar que el estudio de metodologías en seguimiento y evaluación de inversiones, permite proponer una nueva metodología de seguimiento y evaluación que sea una herramienta presupuestal para el uso adecuado de los recursos disponibles, lo cual al unirse con las acotaciones de Castagnola Sanchez (2019), quien investigó el impacto de las políticas públicas de seguridad ciudadana en el distrito de Lima, Perú, destacando que se asignaron recursos logísticos y se aprobaron políticas públicas sociales, con asistencia legal por parte de la municipalidad distrital, logrando el cumplimiento de lo pautado al evaluar las políticas públicas, describiendo escenarios exitosos al ejecutar proyectos de programas sociales tales como vaso de leche, talleres productivos y otras actividades para 
la atención de necesidades de la población. Lo anterior se relaciona con lo descrito por Martinez, Rios y Lavin (2015), quienes establecen la posibilidad de construir modelos predictivos para la gestión, permitiendo disponer de criterios para la conceptualización del proyecto como tal.

Otro aspecto importante del ámbito documental, es el aportado por Calvillo Barrios, J. (2019), quien destaca que desde la metodología cualitativa, se puede proyectar a futuro la necesidad de cambiar los modelos curriculares educativos para ir incentivando el rescate de la construcción de proyectos en la población, a fin que detecten en cada espacio de su vida, debilidades y fortalezas de las gestiones públicas y privadas con criterios de equidad, cobertura y compromiso social. Cedeño León, J \& Sánchez Erazo, A. (2020) resaltan el impacto socioeconómico de la gestión de planeación en Ecuador para financiar proyectos que por las contingencias sanitarias y económicas causadas por el Covid-19, obligando al estado a solicitar financiamiento del Fondo Monetario, destacando que el impacto económico de esta pandemia ha incidido en el financiamiento de proyectos, pues se han cerrados empresas y ha disminuido el caudal tributario recibido por el erario público, impactando negativamente los presupuestos para invertir en nuevos proyectos especiales, requiriendo un apoyo rápido de financiamiento, asesoramiento en política y asistencia.

En el artículo de Sanca Vergara, M. N. \& Cárdenas Saavedra, A. (2021) se comprobó la relación existente entre la gestión y el perfil de los clientes, entendiéndose que cuando se planifica un proyecto, existen ciertas características de los beneficiarios o conglomerado social al cual se dirige, para lo cual incorporaron una Gestión de Relaciones con el Cliente, alcanzando una correlación alta, aplicable también a los proyectos especiales de la gestión pública. Es por ello, que en base a las buenas prácticas se han identificado habilidades del personal decisorio en organizaciones que generan 
éxito, es por ello que Durán (2020), señala que se debe disponer de líderes competentes en la relación y coordinación de equipos de manera eficaz en escenario de trabajos diversos.

De esta forma la Teoría general de sistemas-TGS, según Valencia, S y Zetina, A. (2016), establece un enfoque sistémico que profundiza las características a nivel estructural y funcional de los sistemas, es decir, a nivel de relaciones, interacciones, componentes y subsistemas, en todo su entorno. El cual es clave para el éxito de los proyectos, sobretodo porque desde la conceptualización de estos deben considerar todos los elementos para que en su ejecución no se vean afectados. De hecho Pimenta, C. y Pessoa, M. (2015), desarrolla su teoría basada en la aplicación en modelos de gestión, con una identificación, basada en la aplicación de fenómenos biológicos que visualizan el todo como la suma de sus partes, de allí que su aplicabilidad se extrapoló a distintas áreas del quehacer humano, utilizándolas con éxito. Según señala Chiavenato (2004), la administración actúa como un sistema, entendiendo su basamento en el trabajo conjunto de los niveles decisorios y subordinados, donde se define los objetivos y responsabilidades, los cuales servirán de hitos de control y seguimiento, lo que aplica a la construcción de un proyecto.

De igual manera, para Correa, H. (2015), las responsabilidades para el cumplimiento de los objetivos se permea en todos los niveles de la organización y la planificación estratégica es severamente afectada por la falta de la organización, y conexión entre los sectores responsables, actuando como islas, cumpliendo con sus actividades individuales y no con el enfoque grupal como requiere el enfoque sistémico, asimismo, señala que otro aspecto como punto crítico es la bifurcación entre lo planificado y el presupuesto aprobado, el cual conlleva al incumplimiento de los planes establecidos. se desprende de lo anterior que en el marco de la OCDE para la gobernanza de 
las infraestructuras, planteado por la Organización para la Cooperación y el Desarrollo Económicos (OCDE) (2014) donde se destaca que los proyectos especiales basados en infraestructuras, se ejecutan considerando un impacto a largo plazo y por períodos largos de gestión, por tanto al organizarlos requieren ser previsibles en cuanto a los ciclos políticos y económicos que engloban su ejecución, a fin que los resultados no se vean limitados por estas variables y su incidencia en los sectores beneficiarios.

Los proyecto especiales que responden a necesidades de la población rural o urbana, más allá de ser de infraestructura requieren atender diversos ámbitos, de allí que Vergara Valera, R., Arias Calderón, J. E. \& Rodríguez Vásquez, M. E. (2020) relacionen los proyectos con relación a la solución de la congestión urbana en Santiago de Cali, presentado como un estudio de caso de política Pública, donde destacan que la planeación del desarrollo urbano ha estado distante de atender las necesidades de los colectivos, dejando actuar a la población de forma anárquica, construyendo una situación de saturación, sin acceso a servicios fundamentales de agua, electricidad, salud y educación y congestionando el servicio de transporte urbano en detrimento de la calidad de vida. Focalizando estas ideas, Sánchez Ruiz, J., Larrea Silva, J. y Pardo Villalta, Y. (2021) en el estudio evaluación multicriterio de los atractivos turísticos de la parroquia Jimbura, Cantón Amaluza, Loja-Ecuador 22020, valoraron la metodología de proyectos puesta en práctica por el Ministerio de Turismo, describiendo que son algo subjetivas, partiendo de parámetros cualitativos y cuantitativos guiados de una manera imparcial, lo que resta enfoque científico a los resultados esperados, generando improvisaciones que a lo largo requieren ajuates en la gestión, impactando costos, inversiones y resultados en cuanto a la satisfacción de las necesidades de los conglomerados beneficiarios, conduciendo a que el éxito en el diseño y ejecución de los proyectos dependen de la fiabilidad metodológica que los inspiran. 
Puntualizando algunas debilidades en la construcción de un proyecto Benavente García, J. P. (2021) destaca las nuevas tendencias en la gestión pública en procura de mejorar el bienestar de la población, donde el diagnóstico como parte principal en la etapa de inicio del proyecto especial requiere alcanzar indicadores de brechas de servicios, infraestructura y potencial financiero con la finalidad de incorporar criterios de prioridad y orden que garanticen la ejecución de una inversión no solo acorde con lo pautado en los presupuestos, sino también con las necesidades a resolver para la población de usuarios, garantizando una ejecución y conclusión oportuna, poniendo en práctica la implementación de medidas correctivas en cuanto a acceso y calidad de los servicios o infraestructura. En este mismo enfoque, el aporte de Pérez Rodríguez, C. A. (2021) destaca la importancia de los talentos humanos en cuanto a impregnar la etapa inicial del proyecto con aportes creativos y visionarios, considerados desde el estudio de indicadores no solo de espacios, también de congruencia de las políticas y normativas orientadas a disminuir los impactos negativos, que incidirán en las etapas de desarrollo y cierre del proyecto especial. A propósito de esto, Hernández Madrigal, M. y Ramírez Flores, E. (2013) consideran que la evaluación de los proyectos no es un proceso que se active solamente en el cierre, sino que es transversal a todas las etapas, facilitando valorar índices de calidad, riesgo, percepciones y aprendizajes que guien la toma de decisiones para los ajustes pertinentes.

\section{MATERIALES Y MÉTODOS}

Los aspectos metodológicos asumidos presentan una investigación que se realizará bajo un enfoque cualitativo, para ampliar toda la disponibilidad de información que suma al entendimiento del fenómeno social en la búsqueda de distintas fuentes de información como documentos, comportamientos, eventos u otros, con el fin de universalizar los resultados. La investigación 
documental es una técnica de investigación cualitativa que se encarga de recopilar y seleccionar información a través de la lectura de documentos, libros, revistas, grabaciones, filmaciones, periódicos, bibliografías, etc. Se aborda la categoría de proyecto, y subcategorías: diagnóstico, expediente técnico y evaluación de proyectos, los cuales permitirán identificar aspectos, lo cual a su vez permitirán una visión panorámica de la realidad en función de lo visualizado a futuro cumpliendo con el aspecto predictivo.

Este Proyecto está enmarcado en los lineamientos para la creación, gestión y cierre de programas y proyectos especiales llevando el análisis a través del abordaje de los entrevistados, documentos de la Institución y antecedentes. Muchas personas tienen la idea equivocada de que toda la investigación implica la administración de cuestionarios o hacer experimentos. No obstante, la búsqueda de información se realizó mediante el análisis de documentos que permitieron expandir los elementos del tema asumido como lo es la formulación de proyectos especiales para la inversión pública en función de las necesidades de los colectivos humanos. Cuando se asume una investigación de tipo documental, se piensa en su relación con las ciencias sociales, sin embargo las mismas engloban cualquier rama de la ciencia que ve en las informaciones previas una alternativa de exponer hasta dónde se hay avanzado en el ámbito científico investigado. El modelo presentado por Fernándes, E.,Guimaraes,. D. (2013), toma como referencia los establecidos por el Project Managment Institute (PMI), donde hace énfasis en nueve zonas de conocimiento, como son gestión de integración, del alcance, tiempo. Costo, calidad, de recursos humanos, comunicaciones, riesgo y abastecimiento. 


\section{RESULTADOS}

Los resultados de la revisión documental hasta esta altura del desarrollo de la investigación permiten indicar que un proyecto es el resultado de una problemática de necesidad de un producto, servicio o resultado único, para lo cual se establece un esfuerzo temporal. Desde este punto de vista la forma más sencilla de identificarlo es que se dispone de un inicio y un fin establecido, el cual no necesariamente se culmine al cumplir los objetivos, dado que durante la ejecución varíe su factibilidad o cambie la necesidad preestablecida. Visualizar la afectación de proyectos especiales por una desviación de costos y tiempo en la ejecución del proyecto, debido a la falta de implementación de normas de calidad, resaltando la inapropiada tramitación del proyecto por ausencia de cultura de este tipo de gestiones que influyen en la correcta formulación considerando que la calidad de vida es el factor de mayor ponderación de influencia.

Es por ello que se apreciaron recomendaciones para la implementación de la gestión de proyectos basados en el Instituto de Gestión de Proyectos (Proyect Managment Institute) y así disponer de una estructura fortalecida para una gestión eficaz de los proyectos, alcanzada con la evaluación de aspectos tales como la comparación de resultados de costos beneficios que contribuyan a un mayor valor agregado. También se destaca la utilización de distintas metodologías de evaluación de proyectos como VAN, árboles binomiales y fórmulas de black-scholes, con la finalidad de valorar la relación costo beneficio y su incidencia directa en el valor agregado.

En relación a los proyectos, los aportes de Fernández, E., Guimaraes, D. (2013) establecen la adopción de un modelo de gestión de proyectos, así como, su planificación, aplicado a distintas áreas, y se estructura en nueve (9) ítems o zonas de conocimiento, para ello se visualiza en la siguiente figura el modelo de gestión de proyecto y planificación. Las tendencia y aspectos 
comunes detectados arrojan que existen dos macro bloques para la realización de proyectos, la planificación y la gestión, los cuales como etapas interactúan entre sí, pues la primera conlleva a la segunda y la segunda detecta factores que pudiesen presentarse con aristas distintas y obligan a tomar en cuenta aspectos correctivos, en la etapa de planificación se da inicio a la construcción estratégica del proyecto, pero esto es una propuesta a futuro en el plano de lo ideal, la gestión sin embargo es la puesta en marcha, donde se ejecuta lo planificado, se controla, realimenta y finalmente se concluye con un cierre cuya evaluación orienta la construcción de otros proyectos similares, dando respuesta a las limitaciones detectadas.

De acuerdo a lo detectado, distintos autores coinciden en cuanto a aspectos diversos de la planificación y ejecución de los proyectos, entre ellos Balcazar, R. (2015). Ministerios aplicando obras por impuestos en Perú. Quien establece los mecanismos de financiamientos de proyectos especiales del Estado a través de la canalización de tributos, Camisón, C., C, S. y González, T. (2015), destaca algunas reflexiones para corregir enfoques y funciones, coincidiendo con De la Peña, G. y Velázquez, R. (2018) quienes consideran sobre la teoría general de sistemas y el enfoque sistémico donde las etapas de planificación y ejecución y cada una de las subpartes que la componen, guardan entre sí la integración e intercambio sistémico donde cada elemento es indispensable, con una flexibilidad para corregir sus funciones y alcances para llegar a las metas o dejar recomendaciones para futuras aplicaciones.

Otra particularidad detectada es que de acuerdo a Fernández, M., Seixas, E. \& Carvallo, A. (2019) se describen cinco fases las cuales son asumidas en líneas generales por otros investigadores, de allí que en proyectos especiales del Estado, la fase 1 de aprobación contemple la conceptualización, análisis de variabilidad, selección y aprobación del proyecto, la segunda fase denominada definición, definen los requerimientos, análisis de riesgo, 
propuestas y memorias del proyecto, los tiempos planteados para la futura ejecución y la distribución de recursos, la cuarta fase nombrada como la ejecución, comprende seguimiento y replanificación, gestión de cambios y de incidencias, lo cual da paso a la fase 5 de cierre, donde se entrega la acción lograda, se evalúa y se establece el plan de seguimiento.

\section{DISCUSIÓN}

Han quedado algunos vacíos, aun cuando los criterios epistemológicos arrojan que hay coincidencia en cuanto a la etapa de ejecución con la realización del control, autores como Espinoza, P., Meza, A., Paiva, F. (2018), la plantean ejecutada como meros formalismos y no arrojan políticas contundentes para el seguimiento, con una supervisión que permita informes de progreso, adoptar acciones correctoras a partir de los informes de evaluación, donde se adecuen los recursos disponibles al alcance, aun cuando esto implique el reconocimiento de fallas desde la etapa de planificación.

De lo anterior, al momento de cierre se establecen algunos indicadores que no son considerados por quienes evalúan proyecto, entre otros esta que la emisión de informes finales de evaluación son ajustados para que reflejen las metas que se aspiran y no los logros reales alcanzados, dejando un distanciamiento entre lo que se divulga y lo que los beneficiarios sienten como respuesta a sus necesidades, consideradas en el diagnóstico a inicios de la planificación. Según Baca y Herrera (2016), estos elementos surgen desde el diagnóstico en un análisis de escenarios, de condiciones y limitaciones que revelan las necesidades a abordar para dar satisfacción a la población. De allí que este pendiente el abordaje al final del análisis de actores, de la problemática, de objetivos y de escenarios donde se ve si la solución alcanzada, hasta qué punto resuelve el problema en la realidad y de no ser así, 
resaltar lo pendiente, a que se le atribuyen las fallas y que se debe considerar para mejores aplicaciones.

Es un elemento resaltante lo planteado por Sheilah Miranda, L. (2021) donde un proyecto en sus tres etapas de inicio, desarrollo y cierre debe precisar actividades, recursos, herramientas informáticas, capacidades institucionales, técnicas y normativas como base para el diseño y seguimiento de las estrategias y articulación en la construcción de mecanismos para medir los impactos en cualquiera de las etapas del proyecto, orientando así la toma de decisiones correctivas. Lo planteado se refuerza con los criterios de Serrano Martínez, J. M., García Marín, R. Finisterra, L. (2017) en el enfoque de los proyectos considerando no modelos estándares, sino avocados a las realidades complejas tanto de territorios como de necesidades y posibilidades de financiamiento, dictaminado por políticas públicas de administración de la inversión para evitar sobredotaciones, excesos y accionares poco armoniosos con el gasto público que se dispone. Es así, que Rivera, A. (2019), destaca que los niveles de gobiernos: locales, nacionales e internacionales, deben establecer esfuerzos conjuntos para la disposición de bienes y servicios de calidad, que implique de manera positiva en el desarrollo social. Es importante, la identificación de la importancia de la articulación en los niveles de gobierno para el alineamiento de los objetivos y metas para la disposición del valor público. Las aportaciones de Ruiz Rivadeneira, A. M. (2017) en cuanto a la priorización de inversiones desde la plataforma de experiencias de la Organización para la Cooperación y Desarrollo Económico (OECD), se aprecian problemas comunes que perturban la buena práctica proyectiva, tales como que la inversión no responde realmente a las necesidades, sino que se subvalora la realidad de presupuesto- costo, desligando la inversión pública y privada de los efectos sociales y económicos esperados desde el diseño del proyecto. Tapia Pinto, J. W. (2018) enfatiza en la existencia de factores 
determinantes del éxito en las inversiones del sector público, destacando la innovación como uno de ellos, la viabilidad de este aspecto depende de la identificación de factores productos esperados en base a procesos, reglas, procedimiento, regulaciones, rediseños, capacidades que optimicen la operatividad organizacional .

\section{CONCLUSIONES}

Las conclusiones permiten resumir los hallazgos que en distintos documentos han planteado otros investigadores donde se percibe la coincidencia en la estructuración del plan de proyecto el cual tiene dos macro bloques: uno de planificación y otro de gestión, de lo cual permite considerar que existe la opción de separar en tres bloques, siendo las etapas de inicio, desarrollo y cierre, un esquema general para realizar un proyecto, el primero un bloque conceptual en el cual incluya elementos del diagnóstico de todo lo relacionado con la situación, donde se aprecian las necesidades, se justifica el desarrollo de un proyecto, indicando las metas y los objetivos a lograr, realizando proyecciones de resultados esperados y alcances. De estos elementos conceptuales surge la realización de la etapa de planificación, representada por un plan de acción donde se estipulen las acciones, actividades y tareas, los plazos de realización, los costos y recursos y un cronograma ajustado a todas las tareas, la planificación está formulada en el plano de lo ideal, de allí la segunda etapa es lo concerniente al desarrollo, por eso para la ejecución no se considera la mera realización de tareas, sino el control y la evaluación de las mismas utilizando instrumentos de seguimiento y medición como matrices de productos y resultados, construidas con indicadores surgidos del cotejo de lo planificado con lo percibido en la realidad, derivando cualquier otro instrumento necesario, hojas de cálculo para el control y finalmente el análisis evaluativo que de firmeza a los 
hallazgos y a la toma de decisiones, llegando así a la etapa de cierre, que es ya el proyecto concluido y entregado, donde se destaca lo logrado y se recomienda en función a la realización de nuevas ejecuciones.

También es de considerar que los proyectos especiales, ya surgen en un contexto de diagnóstico las condiciones de los servicios, se evalúa la eficiencia del uso de los recursos basados en la cuantificación de indicadores, orientando la toma de decisiones para nutrir las necesidades de un proyecto, los controles y la evaluación durante y después del desarrollo y en función de los resultados, siempre argumentando en la medición de indicadores relacionados a los costos y beneficios. De allí que quede pendiente profundizar en cuanto al desarrollo de la gestión de calidad involucre inspección, control estadístico, aseguramiento de la calidad e integración, con enfoque del beneficiario, liderazgo, compromiso de las personas, enfoque de procesos, mejora continua, toma de decisiones basadas en evidencia y gestión de las relaciones, las cuales son transversales en la obtención de la calidad en bienes y servicios.

Se especifica así que el proyecto especial, comprende instancias administrativas, organizacionales y gerenciales en función a la planeación y ejecución de actividades a fin de garantizar el logro de los objetivos con monitoreo y control, ejecución implementación, mantenimiento y formulación de los proyectos, bajo un marco legal y la administración interna para gestionar y coordinar todos los recursos, se espera que la culminación de este trabajo de investigación permita detectar los valores de eficiencia y eficacia, para en base a ello desarrollar un marco teórico epistemológico que mejore las acciones y nutra el mundo del conocimiento.

\section{REFERENCIAS}


Aranzamendi, M. (2017) Gestión de proyectos en el programa subsectorial de irrigaciones, Lima. Tesis de Maestría para la obtención del grado de Maestro en Gestión Pública. Universidad Cesar Vallejo. http://repositorio.ucv.edu.pe/handle/20.500.12692/14525

Armendáriz E., Fretes V. (2017). América Latina necesita gastar mejor. Recuperado de https://www.weforum.org/.

Baca, N. y Herrera, F. (2016). Proyectos sociales. Notas sobre su diseño y gestión en territorios rurales. Convergencia, 23(72),69-87. Recuperado en 09 de enero de 2021, de http://www.scielo.org.mx/scielo.php?script=sciarttext\&pid=s140514352016 000300069\&lng=es

Balcazar, R. (2015). Ministerios aplicando obras por impuestos en Perú: la luz al final del túnel. Consultora Llorente \& Cuenc, España.

Benavente García, J. P. (2021) Nuevas Tendencias en la Gestión de la Inversión Pública para la Mejora del Bienestar de la Población Gobierno Regional del Cusco La cartera de inversiones 2019-2021, Invierte.peru. Ministerio de Economía y Finanzas.

Calvillo Barrios, J. (2019) ¿Ser o no ser? La crisis de la educación pública en México. Argumentos y propuestas. Sinergias Educativas, vol. 4, núm 2, 2019. Grupo Compás, Ecuador. Disponible en: http://www.redalyc.org/articulo.oa?id=573561022004. DOI: https//doi.org/10.31876/se.v4i2.40

Camisón, C., Cruz, S. y González, T. (2015). Gestión de la calidad: Conceptos, enfoques, modelos y sistemas. Pearson. Prentice hall. Recuperado de https://porquenotecallas19.files.wordpress.com/2015/08/gestion-de-lacalidad.pdf

Castagnola Sánchez, V. R. (2019) Impacto de las políticas públicas de seguridad ciudadana en un distrito de Lima. Universidad Cesar Vallejo, Lima, 
Perú, castagnolavictor1965@ hotmail.com, ORCID: 0000-0002-3515-6326, https://scholar.google.es/citations?user=UCZIyIQAAAAJ\&hl=es

Cedeño León, J \& Sanchez Erazo, A. (2020) El impacto socioeconómico del Ecuador y su relación con el Fondo Monetario Internacional durante el Covid-19. Jornal of Business and Entreprenurial Studies. ISSN: 2576-0971

Chiavenato, 1. (2004) Introducción a la Teoría General de la Administración. Madrid. Mc Graw Hill.

Correa, H. (2015). Planificación Estratégica para el Desarrollo Regional y Rural. Recuperado de http://cies.org.pe/sites/default/files/investigaciones/001-h.correa. planificación estratégica para desarrollo regional y rural.pdf el 02-08-2016 De la Peña, G. y Velázquez, R. (2018). Algunas reflexiones sobre la teoría general de sistemas y el enfoque sistémico en las investigaciones

Durán, M. Líderes y organizaciones culturalmente diversas, un desafío para el presente siglo. Revista Unimundo, Vol 1 - $\mathrm{N}^{\mathrm{o}}$ 19, 2020. https://revistas.uniminuto.edu/index.php/Pers/issue/view/195

Espinoza, P., Meza, A., Paiva, F.(2018) “Obras por Impuestos, impacto en la población peruana y en la creación de valor de las empresas Caso de estudio, Proyecto: Mejoramiento de los servicios educativos IE. $\mathrm{N}^{\circ} 100$, provincia de Huaral Lima”, Tesis de maestría, Universidad Peruana de Ciencias Aplicadas. Fernándes, E., Guimaraes, D. (2013). Proyectos Educativos y Sociales: Planificación, gestión, seguimiento y evaluación. Madrid: Narcea, S. A. de Ediciones.

Fernandes, M., Seixas, E, \& Carvalho, A. (2019). Para além dos obstáculos: experiencias de consultas públicas e a possibilidade de ética e relevancia na participacao. Comunicacao e Sociedade, 36, 57-80. https://dx.doi.org/10.17231/comsoc.36(2019).2345 
García Reategui, J. H. (2015) Gestión pública y valor público. Lima: Actualidad Gubernamental N 78. Obtenido de https://goo.gl/64vKGG

Hernández Madrigal, M., Ramírez Flores, E. (2013). Evaluación de los proyectos de inversión con beneficios intangibles: Aplicación de modelo Costo-Efectividad en la prevención del cáncer cervicouterino- Ciencia y sociedad 2013; 38(1): 115-140.

http://www.scielo.org.mx/scielo.php?script=sciarttext\&pid=S018845572016 000100232\&tIng=es.

Ley N² 27654 Marco de Modernización de la Gestión de Estado. Lima Perú Lovón, L. (2019). Factores asociados a la ejecución de los proyectos técnicos de inversión pública en la administración de contratos de obra en Electro Sur Este S.A.A.-2018. Tesis doctoral de Gestión Pública y Gobernabilidad. Universidad Cesar Vallejo-Escuela de posgrado.

Martinez, J. Rios, D. y Lavin, J. (2015). Modelos bayesianos de predicción y gestión de tráfico. Tesis doctoral de la Universidad Rey Juan Carlos-España Mattar, J. y Cuervo, L., (2017), Planificación para el desarrollo en América Latina y el Caribe Enfoques, experiencias y perspectivas, para los planes nacionales de desarrollo de Colombia, Ecuador y Brasil. CEPAL-Santiago Munsibay, M. (2019). Análisis de los proyectos de inversión pública del distrito de Sanagorán, provincia de Sánchez Carrión departamento de La Libertad. Tesis doctoral en gestión pública y gobernabilidad. Universidad César Vallejo-Perú.

Organización para la Cooperación y Desarrollo Económico (OCDE, 2014). Marco de la OCDE para la Gobernanza de las Infraestructuras. Disponible en: https://www.oecd.org

Palchisaca, M. (2015). La articulación de la Planificación con el Presupuesto. “Análisis del marco conceptual y de algunas experiencias en América Latina" http://repositorio.iaen.edu.ec/bitstream/24000/3855/2/Caratula\%20y\%200tr 
Pérez Rodríguez, C. A. (2021) Fortalecimiento del semillero gestión del recurso hídrico implementando el aprendizaje basado en proyectos César Arturo Pérez Rodríguez Journal of Business and entrepreneurial April - June Vol. 5 - 2 - 2021 http://journalbusinesses.com/index.php/revista e-ISSN: 2576-0971.

Pimenta, C. y Pessoa, M. (2015). Gestión Financiera Pública en América Latina: La Clave de la Eficiencia y la Transparencia. New York, EE.UU. Banco Interamericano de Desarrollo.

Rivera, A. (2019). Planeación estratégica para el desarrollo sustentable en el sector turístico. Journal Of Business and Entrepreneurial. Ecuador.

Ruiz Rivadeneira, A. M. (2017) Buenas Prácticas en Planificación y Priorización de Inversiones de los países de la OECD. Ministerio de Economía y Finanzas. Invierteperu.com.

Sanca Vergara, M. N. \& Cardenas Saavedra, A. (2012) Custome Relationship Management y captación de clientes en JMA Soluciones, San Isidro 2020. Jornal of Business and Entreprenurial Studies. ISSN: 2576-0971

Sánchez Ruiz, J., Larrea Silva, J., y Pardo Villalta, Y. (2021). Evaluación Multicriterio de los atractivos turísticos de la parroquia Jimbura, Cantón Amaluza, Loja-Ecuador 2020. Jornal of Business and Entreprenurial Studies. ISSN: 2576-0971. April - June Vol. 5 - 2 - 2021 http://journalbusinesses.com/index.php/revista e-ISSN: 2576-0971

Sanz, A. (2015). Vinculación Plan Presupuesto en América Latina. Recuperado de http://repositorio.cepal.org/bitstream/handle/11362/38940/1/S1500812 es. pdf

Sheilah Miranda, L. (2021) Avances y desafíos de la gestión de la inversión pública en el Perú. Dirección General de Inversión Pública del MEF. Invierte.Perú. Ministerio de Edonomía y Finanzas. 
Tapia Pinto, J. W. (2018) Factores Críticos que Determinan el Éxito y la Persistencia de la Innovación Tecnológica de Productos y Procesos en el Sector Público Peruano: Estudio de Caso. Trabajo de Grado de Maestría. Pontificia Universidad Católica del Perú.

Valencia, S. y Zetina, A. (2016). Evaluación de un proyecto de inversión usando opciones reales para diferenciar el aguacate. Estudios sociales (Hermosillo, Son.), 24-25(47), 232-248. Recuperado en 09 de enero de 2021, de

Vera, L. (2015). Factores que contribuyeron a prolongar la duración del proceso de formulación de los estudios de pre inversión (perfil, pre factibilidad y factibilidad) del proyecto de inversión pública denominado "mejoramiento de la atención de las personas con discapacidad de alta complejidad en el instituto nacional de rehabilitación. (Disertación Tesis maestría). Lima: Pontificia Universidad Católica del Perú. Recuperada en: https://tesis.pucp.edu.pe/repositorio/handle/123456789/5908

Vergara Valera, R., Arias Calderón, J. E. \& Rodríguez Vásquez, M. E. (2020). Congestión Urbana en Santiago de Cali, un estudio de caso de política Pública. Territorios 42. Bogotá 2020 pp 1-29 ISSN: 0123-8418. Universidad del Valle. 